\title{
Learning of Simple Conceptual Graphs from Positive and Negative Examples
}

\author{
Sergei O. Kuznetsov \\ All-Russia Institute for Scientific and Technical Information, Moscow, Russia \\ serge@viniti.msk.su \\ Institut für Algebra, Technische Universität Dresden, Dresden, Germany \\ kuznetso@math.tu-dresden.de
}

\begin{abstract}
A learning model is considered in terms of formal concept analysis (FCA). This model is generalized for objects represented by sets of graphs with partially ordered labels of vertices and edges (these graphs can be considered as simple conceptual graphs). An algorithm that computes all concepts and the linear (Hasse) diagram of the concept lattice in time linear with respect to the number of concepts is presented. The linear diagram gives the structure of the set of all concepts with respect to the partial order on them and provides a useful tool for browsing or discovery of implications (associations) in data.
\end{abstract}

\section{Introduction}

In this paper we propose an efficient algorithmic framework for learning simple conceptual graphs and constructing the diagrammatic representation of the space of these graphs, which may be used for solving knowledge discovery problems. We consider a model of learning from positive and negative examples in terms of formal concept analysis (FCA) [14, [5]. FCA proved to be a helpful mathematical tool for various branches of knowledge processing, including conceptual clustering, browsing retrieval [1], and generation of association rules in data mining 11. We show how this model can be extended to data more general than classical contexts. To this end, we give a definition of the closure operation based on an arbitrary semilattice. Classical binary contexts [14] are obtained when semilattice is taken to be a Boolean lattice. As an example we consider a semilattice induced by a set of graphs with partial ordered labels of vertices. These graphs can be interpreted, for example, as molecular graphs or as conceptual graphs [13], [10] without negation and nestedness, i.e., as simple conceptual graphs. We present some results on algorithmic complexity of generating the concept lattice arising from the semilattice on sets of these graphs. The problem of computing the number of all concepts is \#P-complete, however, an algorithm that constructs linear (Hasse) diagram of the concept lattice in time linear with respect to the number of concepts can be proposed. This result improves the quadratic worst-case time bounds for algorithms given in [7], [12], [6], and [1]. 


\section{Learning from Examples in Formal Concept Analysis}

In general terms, the model proposed in [3] is based on the common paradigm of machine learning: given positive and negative examples of a concept, construct a generalization of the positive examples that would not cover any negative example. First, we present a particular case of the model, which can easily be described in terms of FCA. The following definition recalls some well-known notions from [14, [5].

Definition 1. Let $G$ be a set of objects, $M$ be a set of attributes, and $I$ be a relation defined on $G \times M$ : for $g \in G, m \in M, g I m$ holds iff the object $g$ has the attribute $m$, the triple $K=(G, M, I)$ is called a context. If $A \subseteq G, B \subseteq M$ are arbitrary subsets, then the Galois connections are given as follows:

$$
A^{\prime} \rightleftharpoons\{m \in M \mid g I m \text { for all } g \in A\}, \quad B^{\prime} \rightleftharpoons\{g \in G \mid g I M \text { for all } m \in B\} \text {. }
$$

The pair $(A, B)$, where $A \subseteq G, B \subseteq M, A^{\prime}=B$, and $B^{\prime}=A$ is called a concept (of the context $K$ ) with extent $A$ and intent $B$ (in this case we have also $A^{\prime \prime}=A$ and $B^{\prime \prime}=B$ ). The set of attributes $B$ is implied by the set of attributes $A$, or implication $A \rightarrow B$ holds, if all objects from $G$ that have all attributes from the set $A$ also have all attributes from the set $B$, i.e., $A^{\prime} \subseteq B^{\prime} . \diamond$

Now assume that $W$ is a functional (goal) property of objects from a domain under study. For example, in pharmacological applications [3] $W$ can be a biological activity of chemical compounds (like carcinogenicity or, to the contrary, some useful pharmacological activity like sedativity). Thus, $W$ is opposed to the attributes from $M$, which correspond to structural properties of objects. For example, in pharmacological applications the structural attributes can correspond to particular subgraphs of the molecular graphs of chemical compounds.

Input data for learning can be represented by the sets of positive, negative, and undefined examples. Positive examples are objects that are known to have the property $W$ and negative examples are objects that are known not to have this property. Undefined examples are those that are neither known to have the property nor known not to have the property. The results of learning are supposed to be rules used for the classification of undefined examples (or forecast of property $W$ ).

In terms of formal concept analysis, this situation can be described by three contexts: a positive context $K_{+}=\left(G_{+}, M_{+}, I_{+}\right)$, a negative context $K_{-}=$ $\left(G_{-}, M_{-}, I_{-}\right)$, and an undefined one $K_{\tau}=\left(G_{\tau}, M_{\tau}, I_{\tau}\right)$. Here $G_{+}, G_{-}$, and $G_{\tau}$ are sets of positive, negative, and undefined examples, respectively; $M$ is a set of structural attributes; $I_{j} \subseteq G_{j} \times M, j \in\{+,-, \tau\}$, are relations that specify the structural attributes of positive, negative, and undefined examples. Now, a positive hypothesis from [2], 3] (called JSM-hypothesis there) can be defined in the following way.

Definition 2. Consider a positive context $K_{+}=\left(G_{+}, M_{+}, I_{+}\right)$and a negative context $K_{-}=\left(G_{-}, M_{-}, I_{-}\right)$. A pair $\left(e_{+}, i_{-}\right)$is a a positive concept if it is a concept of the context $K_{+}$. If intent $i_{+}$of a positive concept $\left(e_{+}, i_{+}\right)$is not 
contained in the intent of any negative concept (i.e., $\forall g_{-} \in G_{-}, i_{+} \nsubseteq\left\{g_{-}\right\}^{\prime}$ ) and $\left|e_{+}\right| \geq 2$, then the concept $\left(e_{+}, i_{+}\right)$is called a positive hypothesis with respect to the property $W$. Negative hypotheses are defined dually.

Thus, a hypothesis is an implication with a fixed consequent and antecedent equal to the intent of a positive concept. Note that if $\left(e_{+}, i_{+}\right)$is a positive hypothesis with respect to the property $W$, then $i_{+} \rightarrow\{W\}$ is an implication for the context $K_{+-}=\left(G_{+} \cup G_{-}, M \cup\{W\}, I_{+} \cup I_{-} \cup G_{+} \times\{W\}\right)$. Hypotheses can be used for the classification of undefined examples from $G_{\tau}$ (i.e., for forecasting whether they have the property $W$ or not). If an undefined example $g_{\tau} \in G_{\tau}$ has all attributes from the intent $i_{+}$of a positive hypothesis $\left(e_{+}, i_{+}\right)$(i.e., $\left\{g_{\tau}\right\}^{\prime} \supseteq i_{+}$) and does not have all attributes from the intent of any negative hypothesis, then $g_{\tau}$ is classified positively. Negative classifications are defined dually. If $\left\{g_{\tau}\right\}^{\prime}$ does not contain the intent of any negative or positive hypothesis, or includes intents of hypotheses of different signs, then no classification is made.

Example 1. Consider the following sets of positive and negative examples: $G_{+}=$ $\left\{X_{1}, X_{2}, X_{3}, X_{4}\right\}, G_{-}=\left\{Y_{1}, Y_{2}, Y_{3}, Y_{4}\right\}$, and the undefined example $g_{\tau}$, where $\left\{X_{1}\right\}^{\prime}=\{A, B, C\},\left\{X_{2}\right\}^{\prime}=\{A, B, D\},\left\{X_{3}\right\}^{\prime}=\{A, E, F\},\left\{X_{4}\right\}^{\prime}=\{A, C, G\} ;$ $\left\{Y_{1}\right\}^{\prime}=\{A, F, G\},\left\{Y_{2}\right\}^{\prime}=\{A, D, F\},\left\{Y_{3}\right\}^{\prime}=\{B, E, F, G\},\left\{Y_{4}\right\}^{\prime}=\{B, D, F\}$, $\left\{g_{\tau}\right\}^{\prime}=\{A, B, D, E\}$.

The pairs $\left(\left\{X_{1}, X_{2}\right\},\{A, B\}\right),\left(\left\{X_{1}, X_{4}\right\},\{A, C\}\right)$ are positive hypotheses. The pair $\left(\left\{X_{1}, X_{2}, X_{3}, X_{4}\right\},\{A\}\right)$, which is a positive concept with extent larger than one, is not a positive hypothesis, since $\{A\} \subset\left\{Y_{1}\right\}^{\prime},\left\{Y_{2}\right\}^{\prime}$. The negative hypotheses are $\left.\left(\left\{Y_{3}, Y_{4}\right\},\{B, F\}\right),\left(\left\{Y_{2}, Y_{4}\right\}\right),\{D, F\}\right),\left(\left\{Y_{1}, Y_{3}\right\},\{F, G\}\right)$. Since $\{A, B\}$, the intent of the first positive hypothesis is contained in $\{A, B, D, E\}$, the intent of the undefined example, whereas no negative intent does, the undefined example $\left\{g_{\tau}\right\}$ is classified positively. $\diamond$

\section{Extension of the Learning Model}

In this section we use a simple construction from [9], where a partial order on graphs with ordered labels of vertices and edges is completed to a semilattice (actually to a distributive lattice).

Let $\Omega_{g}$ be a set of graphs with partially ordered labels of vertices and edges. For molecular graphs this can correspond to some natural hierarchy of classes of chemical elements.

Suppose that two graphs $F=\left\langle\left\langle V_{F}, M_{F}\right\rangle, E_{F}\right\rangle$ and $H=\left\langle\left\langle V_{H}, M_{H}\right\rangle, E_{H}\right\rangle$ from $\Omega_{g}$ are given. Here $V_{F}, V_{H}$ are sets of vertices, $M_{F}, M_{H}$ are sets of vertex labels, $E_{F}, E_{H}$ are sets of edges, respectively, $E_{F} \subseteq V_{F} \times V_{F} ; E_{H} \subseteq V_{H} \times V_{H}$. The sets $m_{F}$ and $m_{H}$ belong to a set of labels ordered with respect to some ordering $\leq$. We shall consider only vertex labeling. The case with labeled edges and vertices can be reduced to the case where only vertices are labeled.

Definition 3. $F$ subsumes $H$ or $H \preceq F$ iff there exists one-to-one mapping $\varphi$ from the set $V_{H}$ into the set $V_{F}$ that maps each vertex $v_{H} \in V_{H}$ with label $m_{H}$ to a vertex $v_{F} \in V_{F}$ with label $m_{F}$ such that $m_{H}\left(v_{H}\right) \leq m_{F}\left(v_{F}\right)$. The mapping should not violate incidence relation, i.e. if $(a, b) \in E_{H}$, then $(\varphi(a), \varphi(b)) \in E_{F}$. $\diamond$ 
The graphs of the aforementioned form can be interpreted as conceptual graphs [13], and $\preceq$ as the specialization relation [10]. The relation $\preceq$ is a generalization of the "subgraph isomorphism" relation and coincides with it when labels are not ordered.

Definition 4. Let $\mathcal{H}=\left\{H_{1}, \ldots, H_{n}\right\}$ and $H_{1}, \ldots, H_{n} \in \Omega_{g}$. Then $\mathrm{N}(\mathcal{H}) \rightleftharpoons$ $\left\{H_{i} \mid \forall H_{j} \in \mathcal{H}, H_{i} \preceq H_{j} \rightarrow H_{j}=H_{i}\right\}$.

For $i \neq j\left\{H_{i}\right\} \sqcap\left\{H_{j}\right\} \rightleftharpoons \mathrm{N}\left(\left\{H: H \preceq H_{i}, H \preceq H_{j}\right\}\right)$ (the set $\left\{H_{i}\right\} \sqcap\left\{H_{j}\right\}$ consists of all graphs maximal by inclusion among those subsumed by both $H_{i}$ and $H_{j}$.

Let $\mathcal{H}=\left\{H_{1}, \ldots, H_{n}\right\}$ and $\mathcal{F}=\left\{F_{1}, \ldots, F_{m}\right\}$ be sets of graphs from $\Omega_{g}$. Then $\mathcal{H} \sqcap \mathcal{F}=\mathrm{N}\left(\cup_{i, j}\left\{H_{i}\right\} \sqcap\left\{F_{j}\right\}\right) . \diamond$

Example 2. Let $F_{1}$ and $F_{2}$ be molecular chemical graphs given in Fig. 1.
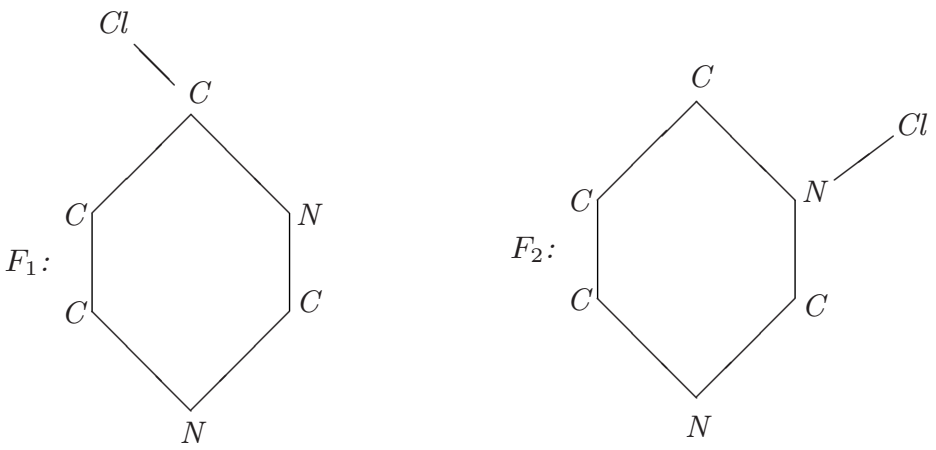

Fig.1

The vertex labels of the graphs are ordered as follows ( $x$ denotes "an arbitrary chemical element"): $x \leq C, x \leq N, x \leq C l$, the labels $C, N$, and $C l$ are incomparable. Then $\left\{F_{1}\right\} \sqcap\left\{F_{2}\right\}=\left\{H_{1}, H_{2}, H_{3}\right\}$, where $H_{1}, H_{2}$, and $H_{3}$ are given in Fig. 2.

$C l$.
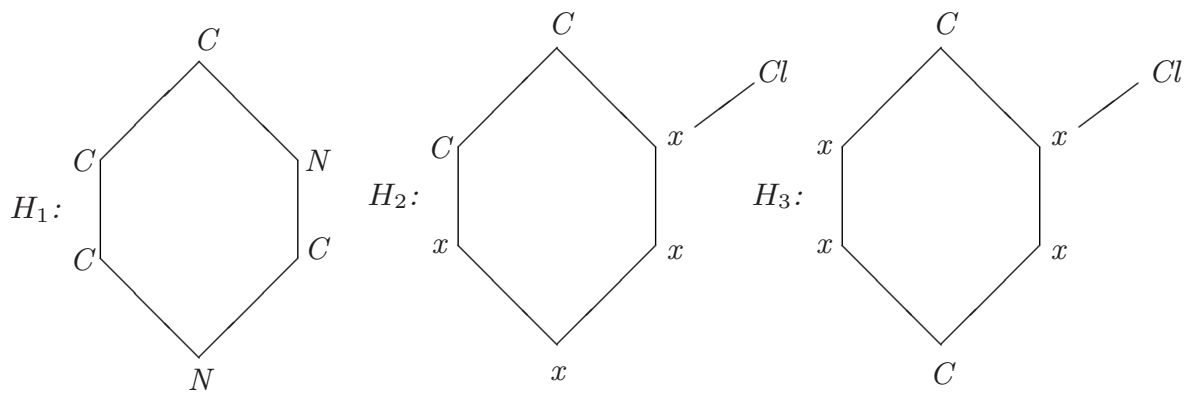

Fig.2 
Here, the disconnected graph $H_{1}$ contains more information about the cyclic structure, whereas $\mathrm{H}_{2}$ and $\mathrm{H}_{3}$ contain more information about the connection of the cycle with the vertex labeled by " $\mathrm{Cl}$ ".

It is easily seen that operation $\Pi$ induces a semilattice $\Omega_{g}^{N}$ with the set of generators $\Omega_{g}$ (i.e., $\sqcap$ is idempotent, commutative, and associative on $\Omega_{g}^{N}$ ). Thus, the order relation $\sqsubseteq$ can be defined as usual: $X \sqsubseteq Y \rightleftharpoons X \sqcap Y=X$. Now we define operations ' and " analogous to those from Section 2.

Definition 5. Let $H_{1}, \ldots, H_{k} \in \Omega_{g}$, then

$$
\begin{gathered}
\left\{H_{1}, \ldots, H_{k}\right\}^{\prime} \rightleftharpoons\left\{H_{1}\right\} \sqcap \ldots \sqcap\left\{H_{k}\right\}, \\
\left\{H_{1}, \ldots, H_{k}\right\}^{\prime \prime} \rightleftharpoons\left\{H \in \Omega_{g}:\{H\} \sqsupseteq\left\{H_{1}\right\} \sqcap \ldots \sqcap\left\{H_{k}\right\}\right\} .
\end{gathered}
$$

It can be shown that " is a closure operation on $\Omega_{g}^{N}$ (i.e., it is extensive, idempotent, and monotone). For arbitrary $X_{1}, \ldots, X_{k} \in \Omega_{g}$ the pair $\left(\left\{X_{1}, \ldots\right.\right.$, $\left.\left.X_{k}\right\}^{\prime},\left\{X_{1}, \ldots, X_{k}\right\}^{\prime \prime}\right)$ is called a concept with extent $\left\{X_{1}, \ldots, X_{k}\right\}^{\prime \prime}$ and intent $\left\{X_{1}, \ldots, X_{k}\right\}^{\prime}$.

The closure operator " and concept from Definition 1 can be obtained from Definition 5 when $\Omega_{g}$ is a set of objects $G, \Omega_{g}^{N}$ is the set of attributes $M, \sqcap$ is the set-theoretic intersection $\cap$ defined on subsets of attributes $M$, the terms $H, H_{1}, \ldots, H_{k}$ stay for objects from $G$ and $\{H\},\left\{H_{1}\right\}, \ldots,\left\{H_{k}\right\}$ stay for their intents. Note that the operation $\sqcap$ and the corresponding " can be defined in lines of Definitions 4-5 for arbitrary partial orders (and thus, data types), not only for those given in Definition 3.

\section{Algorithms and Complexity}

A crucial problem here is that of generation of all concepts of a given context. It is difficult not only to generate the set of all concepts, whose size can be exponential in the size of the source context, but also to calculate or even estimate its size, since the problem of computing all concepts is \#P-complete 8].

Now we describe an algorithm similar to that from [4], which generates the set of all concepts. We transform it in an algorithm generating Hasse diagram in time linear with respect to the number of all concepts.

Let the type of objects and the corresponding closure operator " be fixed (they can be either from Definition 1 or from Definition 5). Below, for definiteness sake, we shall speak in terms of Definition 1, however, all the formulations are the same for $\Omega_{g}$ and the operations ' and "from Definition 5. We assume that objects from $G$ are numbered, and therefore, a set $X \subseteq G$ can be represented by a correspondingly ordered tuple. The numbering of objects from $G$ induces lexicographical ordering of sets from $\mathcal{P}(G)$, the powerset of $G$.

Definition 6. A path is defined inductively as follows:

(1) If $g \in G$ and $\{g\}^{\prime \prime}=\{g\} \cup Z, g \notin Z, Z \subseteq G$, then $[(\emptyset,\{g\}) Z]$ is called a path and $\{g\}^{\prime \prime}$ is called the extent of the path or $\operatorname{Ext}[(\emptyset,\{g\}) Z]$. We also say that $[(\emptyset,\{g\}) Z]$ is an inference of $\{g\}^{\prime \prime}$. The inference $[(\emptyset,\{g\}) Z]$ of $\{g\}^{\prime \prime}$ is canonical (or the path $[(\emptyset,\{g\}) Z]$ is canonical) iff the numbers of all objects from $Z$ (in the sense of numbering of objects from $G$ ) are greater than the number of $g$. 
(2) If $Y$ is a path, $h \in G,(\operatorname{Ext}(Y) \cup\{h\})^{\prime \prime}=\operatorname{Ext}(Y) \cup\{h\} \cup Z, Z \cap Y=\emptyset$, then $[(Y,\{h\}) Z]$ is a path. $\operatorname{Ext}[(Y,\{h\}) Z] \rightleftharpoons(\operatorname{Ext}(Y) \cup\{h\})^{\prime \prime}=\operatorname{Ext}(Y) \cup\{h\} \cup Z$. We say that $[(Y,\{h\}) Z]$ is an inference of $(\operatorname{Ext}(Y) \cup\{h\})^{\prime \prime}$. The inference $[(Y,\{h\}) Z]$ of $(\operatorname{Ext}(Y) \cup\{h\})^{\prime \prime}$ is called canonical iff $Y$ is a canonical path and the numbers of all objects from $Z$ are greater than the number of $h$. $\diamond$

The following procedure (we call it Close-by-One or CbO Algorithm) is based upon the depth-first strategy, though other strategies are possible as well. $Y$ denotes the path to the current concept.

\section{Algorithm 1.}

Step 0. There is only one root vertex where all objects are unlabeled, $Y:=\emptyset$.

Step 1. The current vertex corresponds to the concept with the extent $Y$. The first unlabeled object from $G$, say $X_{i}$, is taken and labeled at $Y,(\operatorname{Ext}(Y) \cup$ $\left.\left\{X_{i}\right\}\right)^{\prime}$ and $\left(Y \cup\left\{X_{i}\right\}\right)^{\prime \prime}=\left(\operatorname{Ext}(Y) \cup\left\{X_{i}\right\} \cup Z\right.$ are computed. A new vertex that corresponds to $\left(\operatorname{Ext}(Y) \cup\left\{X_{i}\right\}\right)^{\prime \prime}$ is generated and connected to the vertex associated with $Y$.

Step 2. If $Z$ contains objects with numbers less than $i$ (i.e., the path $\left[\left(Y, X_{i}\right) Z\right]$ is not canonical), then we label all objects from $G$ at the vertex $\left(\operatorname{Ext}(Y) \cup\left\{X_{i}\right\}\right)^{\prime \prime}$ (thus, the branch will not be extended). If $Z$ does not contain objects with numbers less than $i$ (i.e., $\left[\left(Y, X_{i}\right) Z\right]$ is canonical), then we label all objects from $\left(\operatorname{Ext}(Y) \cup\left\{X_{i}\right\}\right)^{\prime \prime} \cup\left\{X_{1}, \ldots, X_{i-1}\right\}$ at the vertex $\left(\operatorname{Ext}(Y) \cup\left\{X_{i}\right\}\right)^{\prime \prime}$.

Step 3. If all elements of $G$ are labeled at $\left(\operatorname{Ext}(Y) \cup\left\{X_{i}\right\}\right)^{\prime \prime}$, we go to Step 4 . Otherwise, $Y:=\left[\left(Y, X_{i}\right) Z\right]$, and we return to Step 1 .

Step 4. We backtrack the tree upwards to the nearest vertex with unlabeled elements of $G$. If such a vertex exists and corresponds, say, to the path $Z$, then $Y:=Z$ and we go to Step 1 . If such a vertex does not exist, then this means that all concepts have been generated and the algorithm halts. $\diamond$

Example 3. Consider objects $X_{1}, X_{2}, X_{3}, X_{4}$ from Example 1 . In this case, Algorithm 1 constructs the tree with the following left-most branch: root $-\left[\left(X_{1}\right) X_{2}\right]$ $-\left[\left(\left[\left(X_{1}\right) X_{2}\right] X_{3}\right) X_{4}\right]$, which consists of two non-root vertices. Both these vertices are canonical. $\diamond$

Theorem 1. The tree output by Algorithm 1 has $O(|G||L|)$ vertices. The canonical vertices of this tree are in one-to-one correspondence with the concepts. The time complexity of Algorithm 1 is $O((\alpha+\beta|G|)|G||L|)$ and its space complexity is $O((\gamma|G||L|))$, where $\alpha$ is time needed to perform $\sqcap$ operation and $\beta$ is time needed to test $\sqsubseteq$ relation and $\gamma$ is the space needed to store the largest object from $\Omega_{g}^{N}$. When contexts and concepts are given by Definition 1, the time complexity is $\left(|M||G|^{2}|L|\right)$ and the space complexity is $O(|M||G||L|) . \diamond$

In general, the computation of $\sqcap$ and $\sqsubseteq$ is NP-hard, but in some realistic cases it may be polynomial [10.

The lattice order agrees with that of the tree constructed by Algorithm 1 (called $\mathrm{CbO}$ tree), however the corresponding incidence relation (that defines edges of the Hasse diagram) does not agree with the incidence relation in the tree. To construct the Hasse diagram of the lattice, we need to connect each pair of vertices in the tree that correspond to adjacent vertices in the diagram. To this end, we run the following algorithm in the depth-first left-most order. 


\section{Algorithm 2}

Step 0. We are in the root vertex of the CbO tree constructed by Algorithm 1. $Y:=\emptyset, \operatorname{Fr}(C):=\emptyset$, and $\operatorname{To}(C):=\emptyset$ for all concepts $C$. All vertices are unlabeled. Step 1. The current canonical vertex corresponds to the concept with the extent $Y$. For each element $X_{i}$ of $G$ we compute $\left(Y \cup\left\{X_{i}\right\}\right)^{\prime}$ and $\left(Y \cup\left\{X_{i}\right\}\right)^{\prime \prime}$. Among sets $\left(Y \cup\left\{X_{i}\right\}\right)^{\prime \prime}, i=1, \ldots,|G|$, we select those minimal by inclusion. These are extents of concepts adjacent to $\left(Y, Y^{\prime}\right)$ from below. We denote the set of these extents by $M(Y) \cdot \operatorname{Fr}\left(Y^{\prime}\right):=\left\{\left\langle\left(Y, Y^{\prime}\right),\left(Z, Z^{\prime}\right)\right\rangle \mid Z \in M(Y)\right\}$. Thus, the set of arcs in the Hasse diagram leading from the vertex $\left(Y, Y^{\prime}\right)$ to its children is constructed. We label the vertex $Y$ and number the elements of $M(Y)$ using the numbering of $G$ ).

Step 2. For every extent $E \in M(Y)$ we take the corresponding concept $\left(E, E^{\prime}\right)$ and find its canonical inference. This is equivalent to finding the corresponding canonical path in the tree generated by Algorithm 1. We update the set of arcs leading to $\left(E, E^{\prime}\right)$ by letting $\operatorname{To}\left(E^{\prime}\right):=\operatorname{To}(E) \cup\left\{\left\langle\left(E, E^{\prime}\right),\left(Y, Y^{\prime}\right)\right\rangle\right\}$.

Step 3. If there are unlabeled canonical vertices corresponding to extents in $M(Y)$, we pass from $Y$ to the first of them (with respect to the numbering on elements of $M(Y)$ given in Step 2), say $Z, Y:=Z$ and return to Step 1 . If there are no such vertices and $Y$ is not the root of the tree, we backtrack to the parent of $Y$ in the tree, denote it by $R(Y), Y:=R(Y)$ and return to Step 3. If $Y$ is the root of the tree and there are no unlabeled vertices corresponding to extents in $M(Y)$, then algorithm halts. $\diamond$

For every concept $C$ Algorithm 2 outputs sets $\operatorname{Fr}(C)$ and $\operatorname{To}(C)$ of arcs that lead to concepts immediately adjacent to $C$ in the Hasse diagram from above and below, respectively. Thus, the $\mathrm{CbO}$ tree, together with sets $\operatorname{Fr}(C)$ and $\operatorname{To}(C)$ related to each canonical vertex is a representation of the concept lattice. Unlike the incidence matrix of a lattice, whose size is quadratic in the number of concepts, the size of this structure and time needed to construct it are linear. Given also a negative context at the input, one can generate hypotheses by slightly modifying Algorithm 2: at Step 3 it should also be tested whether positive intents are not subsumed by negative examples. The algorithm is also easily extended to include a test for sufficient number of examples supporting a hypothesis, for example, in lines of [1].

Theorem 2. Algorithm 2 constructs the Hasse diagram of a concept lattice in $O\left(\left(\alpha|G|+\beta|G|^{2}\right)|L|\right)$ time and $O((\gamma|G||L|)$ space, where $|L|$ is the number of concepts, $\alpha$ is the time needed to perform $\sqcap$ operation, $\beta$ is the time needed to test $\sqsubseteq$ relation, and $\gamma$ is the space needed to store the largest object from $\Omega_{g}^{N}$. When contexts and concepts are given by Definition 1 the diagram is constructed in $O\left(|M||G|^{2}|L|\right)$ time and $O(|M||G||L|)$ space.

\section{Conclusion}

We presented a learning model in a version of the formal concept analysis that allows processing graph structures. For example, this model can be used for learning implications on simple conceptual graphs [13] or molecular graphs. The 
model can also be extended to arbitrary data structures with partial order. Algorithmic analysis was provided. Though computations on graphs may be hard in general, this does not affect the linear dependence of time and space needed for the computation on the number of resulting concepts (hypotheses, implications).

\section{Acknowledgments}

This work was supported by the Russian Foundation for Basic Research, project no. 98-06-80191 and the Alexander von Humboldt Foundation.

\section{References}

1. Carpineto, C., Romano, G.: A Lattice Conceptual Clustering System and Its Application to Browsing Retrieval. Machine Learning 24 (1996) 95-122

2. Finn, V.K.: On Machine-Oriented Formalization of Plausible Reasoning in the Style of F. Backon-J. S. Mill. Semiotika Informatika 20 (1983) 35-101 [in Russian]

3. Finn, V.K.: Plausible Reasoning in Systems of JSM Type. Itogi Nauki i Tekhniki, ser. Informatika 15 (1991) 54-101

4. Ganter, B: Algorithmen zur Formalen Begriffsanalyse. In: Ganter, B., Wille, R., and Wolff, K. E. (eds.): Beiträge zur Begriffsanalyse. B. I. Wissenschaftsverlag, Mannheim (1987) 241-254

5. Ganter, B., Wille, R.: Formal Concept Analysis. Mathematical Foundations. Springer-Verlag, Berlin Heidelberg New York (1999)

6. Godin, R., Missaoui, R., Alaoui, H.: Incremental Concept Formation Algorithms Based on Galois (Concept) Lattices. Computational Intelligence 11(2) (1995) 246267

7. Guénoche, A.: Construction du treillis de Galois d'une relation binaire. Math. Sci. Hum. 95 (1990) 5-18

8. Kuznetsov, S.O.: Interpretation on Graphs and Algorithmic Complexity Characteristics of a Search of Specific Patterns. Autom. Document. Math. Ling. 23(1) (1989) 37-45

9. Kuznetsov, S.O.: JSM-method as a Machine Learning System. Itogi Nauki Tekhn., ser. Informat. 15 (1991) 17-54 [in Russian]

10. Mugnier, M.L.: On Generalization/Specialization for Conceptual Graphs. J. Exp. Theor. Artif. Intel. 7 (1995) 325-344

11. Pasquier, N., Bastide, Y., Taouil, R., Lakhal, L.: Pruning Closed Itemset Lattices for Association Rules. In: Proc. 14th BDA Conference on Advanced Databases (BDA'98). Hamammet (1998) 177-196

12. Skorsky, M.: Endliche Verbände - Diagramme und Eigenschaften. Shaker, Aachen (1992)

13. Sowa, J.F.: Conceptual Structures - Information Processing in Mind and Machine. Addison-Wesley, Reading (1984)

14. Wille, R.: Restructuring Lattice Theory: an Approach Based on Hierarchies of Concepts. In: Rival, I. (ed.): Ordered Sets. Reidel, Dordrecht Boston (1982) 445470 